\title{
A new candidate supernova remnant in Cygnus
}

\author{
F. Mavromatakis ${ }^{1}$ and R. G. Strom ${ }^{2,3}$ \\ 1 University of Crete, Physics Department, PO Box 2208, 71003 Heraklion, Crete, Greece \\ 2 ASTRON, Radiosterrenwacht, Postbus 2, 7990 AA Dwingeloo, The Netherlands \\ 3 Astronomical Institute, University of Amsterdam, The Netherlands
}

Received 17 July 2001 / Accepted 9 November 2001

\begin{abstract}
Deep optical CCD imaging and spectroscopic observations of four new nebular structures have been performed for the first time. Filamentary and diffuse emission is detected in this field located to the north-east of the CTB 80 supernova remnant (SNR). Two long filaments are discovered to the north of LBN 156, while a $23^{\prime}$ long filament, emitting strongly in the [O III] line, is present to the east of LBN 156. A complex and compact network of filaments is located close to the center of our field. Finally, the last new source of line emission detected in this field is mainly diffuse and patchy and its morphology displays a semi-circular shape. The long-slit spectra of these structures indicate emission from shock-heated gas and the observed variations in the [O III] fluxes most likely reflect differences in the shock velocities. Weak radio emission at $4850 \mathrm{MHz}$ seems correlated with almost all of the new structures. It is proposed that all these structures, with the possible exception of the brightest one, are part of a single supernova remnant. Detailed radio observations should allow the determination of the nature of the radio emission and provide a crucial test of our suggested intepretation.
\end{abstract}

Key words. ISM: general - ISM: supernova remnants

\section{Introduction}

A source of extended radio emission in the Galaxy is usually considered to be a candidate supernova remnant if the radio spectrum is non-thermal, as its origin may be synchrotron emission. Optical and/or X-ray observations can contribute to its positive identification as a remnant, provided that emission is detected and is not absorbed by the interstellar medium. At optical wavelengths significant attenuation due to interstellar extinction allows only relatively nearby remnants to be observable. It is well known that the interstellar medium is far from uniform and homogeneous. Cavities can be found where the density is much lower than the ambient density, as well as regions of much higher density, known as interstellar "clouds". Optical emission from middle-aged remnants is produced by the interaction of the main blast wave with these interstellar clouds. Observations with optical telescopes through narrow-band interference filters e.g. $\mathrm{H} \alpha$, [S II], [O III], and/or long-slit spectra can provide us with information about the shock speed, the electron density and temperature and possible interactions with the local interstellar medium (e.g. Osterbrock 1989; Cox \& Raymond 1985). Wide field imaging offers the additional advantage of studying the morphogology in each emission

Send offprint requests to: F. Mavromatakis,

e-mail: fotis@physics.uoc.gr line and searching for correlations with emission at radio, $\mathrm{X}$-ray and infrared wavelengths.

During deep optical observations of the supernova remnant CTB 80 by Mavromatakis et al. (2001, their Fig. 1), two faint, thin arcs were discovered at the north-east edge of the field of view. Their extent, shape and faintness motivated us to perform new deep observations of the area to the east of the two arcs. In this paper we present deep CCD images of this field in major optical lines. Information about the observations and the data reduction is given in Sect. 2. In Sects. 3 and 4 we present the results of our imaging observations as well as results from long-slit spectra taken at specific locations of interest. In Sect. 5 we report on the results of a search for emission in radio, X-ray and far-infrared wavelengths. Finally, in Sect. 6 we discuss the physical properties of the structures discovered by us.

\section{Observations}

\subsection{Optical images}

The observations presented here were performed with the $0.3 \mathrm{~m}, \mathrm{f} / 3.2$ wide field telescope at Skinakas Observatory on July 28, 29, and 30, 2000. A $1024 \times 1024$ Site CCD was used to image the field of interest, at an image scale of $5^{\prime \prime}$ per pixel, in conjunction with interference filters isolating the emission lines of $\mathrm{H} \alpha$ and $[\mathrm{NII}], \mathrm{H} \beta$, [S II], 


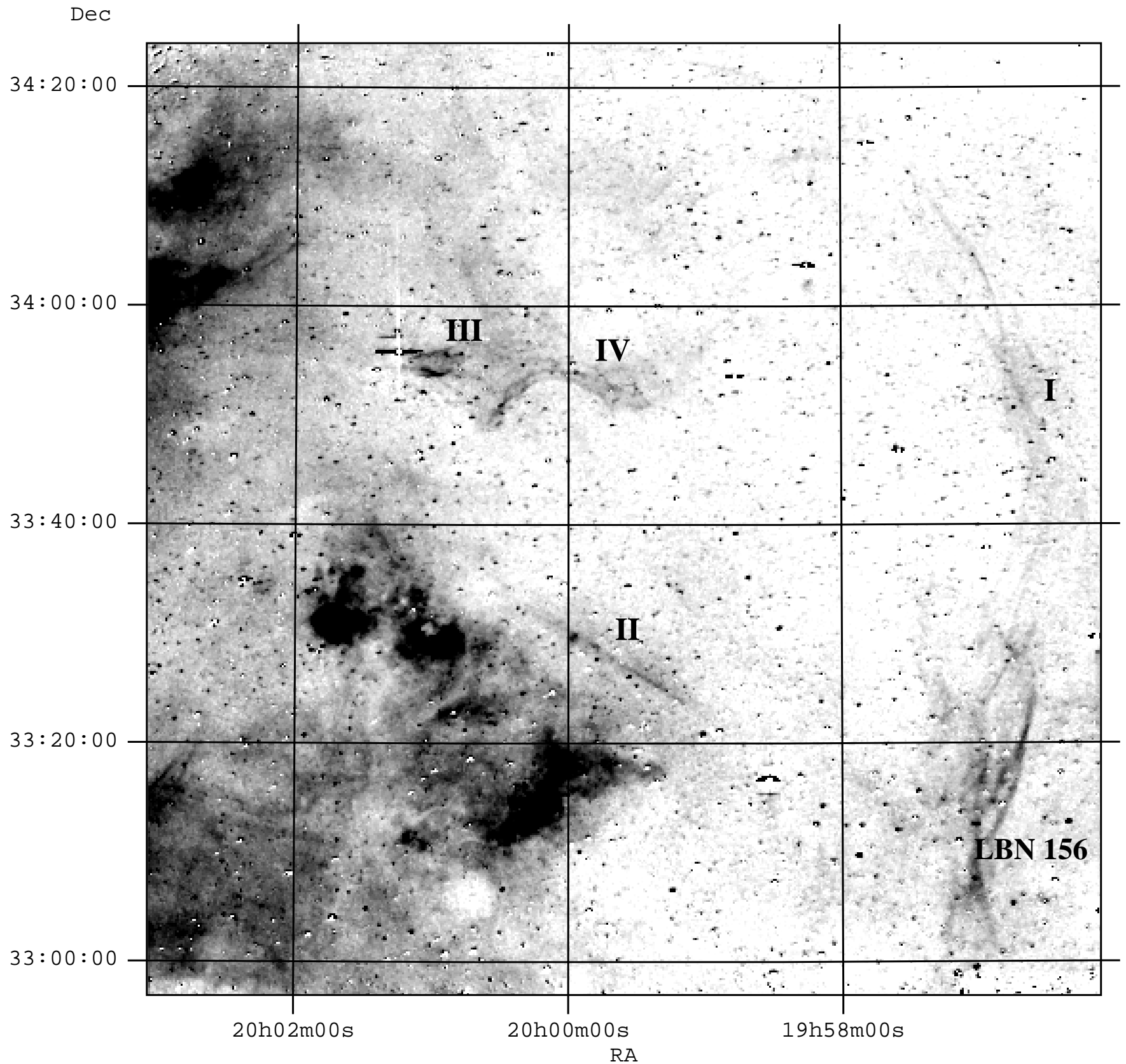

Fig. 1. The field around the candidate remnant in the $\mathrm{H} \alpha+[\mathrm{N}$ II] filter. The image has been smoothed to suppress the residuals from the imperfect continuum subtraction. Shadings run linearly from 0 to $80 \times 10^{-17} \mathrm{erg} \mathrm{s}^{-1} \mathrm{~cm}^{-2} \mathrm{arcsec}^{-2}$. The line segments seen near overexposed stars in this figure and the next figures are due to the blooming effect.

[O II] and [O III]. The major characteristics of the filters can be found in Mavromatakis et al. (2001). The number of frames taken in each filter and the total exposure time are given in Table 1. The final images in each filter are the average of the individual frames. All coordinates quoted in this work refer to epoch 2000.

Standard IRAF and MIDAS routines were used for the reduction of the data. Individual frames were bias subtracted and flat-field corrected using well exposed twilight flat-fields. The spectrophotometric standard stars HR 5501, HR 7596, HR 7950, HR 8634, HR 9087 and HR 718 were used for flux calibration.

\subsection{Optical spectra}

Long-slit spectra were obtained on August 02 and 03, 2000 using the $1.3 \mathrm{~m}$ Ritchey-Cretien telescope at Skinakas Observatory. The spectrophotometric standard stars HR 718, HR 5501, HR 9087, and HR 7596 were used for absolute flux calibration. The data were taken with a 1300 line $\mathrm{mm}^{-1}$ grating and a $800 \times 2000$ Site CCD having a $15 \mu \mathrm{m}$ pixel size and cover the range of $4750 \AA-6815 \AA$. The slit had a width of 7 '. 7 and, in all cases, was oriented in the north-south direction. The number of available spectra from each filamentary structure, and the exposure time of 
Table 1. Log of the exposure times.

\begin{tabular}{lllll}
\multicolumn{5}{c}{ Images } \\
\hline $\mathrm{H} \alpha+[\mathrm{N} \mathrm{II}]$ & {$[\mathrm{S} \mathrm{II}]$} & {$[\mathrm{O} \mathrm{III}]$} & $\mathrm{H} \beta$ & {$[\mathrm{O} \mathrm{II}]$} \\
\hline $4200^{\mathrm{a}}(2)^{\mathrm{b}}$ & $4200(2)$ & $10200(5)$ & $10200(5)$ & $4800(2)$ \\
\hline \hline \multicolumn{5}{c}{ Spectra } \\
\hline area I & area II & area III & area IV \\
\hline $7200(2)$ & $7200(2)$ & $3600(2)$ & $5400(2)$ \\
\hline
\end{tabular}

${ }^{\text {a }}$ Total exposure times in $\mathrm{s}$.

b Number of individual frames.

Table 2. Typical fluxes from imagery.

\begin{tabular}{lllll}
\hline & area I & area II & area III & area IV \\
\hline $\mathrm{H} \alpha+[\mathrm{N} \mathrm{II}]$ & 43 & 64 & 114 & 75 \\
\hline$[\mathrm{S} \mathrm{II}]$ & 11 & 4 & 36 & 20 \\
\hline $\mathrm{H} \beta$ & 2.6 & 11 & 16 & 8 \\
\hline$[\mathrm{O} \mathrm{III}]$ & 1.6 & 16 & 19 & 4 \\
\hline$[\mathrm{O} \mathrm{II}]$ & 22 & 33 & 96 & 37 \\
\hline
\end{tabular}

Fluxes in units of $10^{-17} \mathrm{erg} \mathrm{s}^{-1} \mathrm{~cm}^{-2} \operatorname{arcsec}^{-2}$.

Table 3. Coordinates of slit centers.

\begin{tabular}{lllll}
\hline & area I & area II & area III & area IV \\
\hline$\alpha^{\mathrm{a}}$ & $19^{\mathrm{h}} 56^{\mathrm{m}} 46^{\mathrm{s}}$ & $19^{\mathrm{h}} 59^{\mathrm{m}} 30^{\mathrm{s}} .4$ & $20^{\mathrm{h}} 00^{\mathrm{m}} 58^{\mathrm{s}} .2$ & $20^{\mathrm{h}} 00^{\mathrm{m}} 24^{\mathrm{s}} .6$ \\
\hline$\delta^{\mathrm{a}}$ & $33^{\circ} 57^{\prime} 15^{\prime \prime}$ & $33^{\circ} 26^{\prime} 59^{\prime \prime}$ & $33^{\circ} 54^{\prime} 34^{\prime \prime}$ & $33^{\circ} 52^{\prime} 21^{\prime \prime}$ \\
\hline
\end{tabular}

${ }^{\text {a }}$ Equatorial coordinates for epoch 2000.

each spectrum are given in Table 1. Standard IRAF routines were employed for the preparation and reduction of the long-slit spectra.

\section{The results}

\subsection{The $H \alpha+[N 川]$ and [S II] images}

The field in $\mathrm{H} \alpha+[\mathrm{NII}]$ is dominated by several bright $\mathrm{H}$ II regions, mainly found in the east (Fig. 1). We also find LBN 156 at the south-west edge of our field (Lynds 1965). The two arcs seem to emerge from the northern areas of LBN 156 and extend up to a declination of $34^{\circ} 24^{\prime}$ (area I in Fig. 1). These arcs seem to define rather well part of a circle's periphery, but not centered in the middle of all the new structures, and with a radius of $\sim 42^{\prime}$. Three more new structures are present in this field. The first appears as a $\sim 11^{\prime}$ long filament in the south (area II in Fig. 1). Its center is approximately at $\alpha \simeq 19^{\mathrm{h}} 59^{\mathrm{m}} 34^{\mathrm{s}}$ and $\delta \simeq 33^{\circ} 26^{\prime} 53^{\prime \prime}$, a few arcminutes to the north of Sh 2-99, Sh 2-100 (Sharpless 1959).

The second structure (area III in Fig. 1) is located at $\alpha \simeq 20^{\mathrm{h}} 01^{\mathrm{m}} 00^{\mathrm{s}}$ and $\delta \simeq 33^{\circ} 54^{\prime} 46^{\prime \prime}$, very close to the bright, variable star HD 189918. It consists of two substuctures oriented in the east-west direction and occupies an angular extent of $4.8 \times 2 ! 5$. Optical emission from this area is the brightest among the new structures in this field
(Table 2). Finally, the last new structure appears as a partial arc convex to the north with a radius of curvature of $6 ! 8$ (area IV in Fig. 1). The center of curvature is estimated at $\alpha \simeq 20^{\mathrm{h}} 00^{\mathrm{m}} 01^{\mathrm{s}}$ and $\delta \simeq 33^{\circ} 42^{\prime} 20^{\prime \prime}$. The eastern half of this arc is brighter than the western half.

The morphology of the [S II] image is generally similar to, though not as sharp as, that of the $\mathrm{H} \alpha+[\mathrm{N}$ II] image and is not shown here.

We also note that we have examined the published galactic plane survey of Parker et al. (1979). While the scale and quality of the published images may limit the visualization of weak emission, we find no evidence for any of the emission seen here in $\mathrm{H} \alpha+[\mathrm{N}$ II] and [S II] (or in $[\mathrm{O} \mathrm{III}])$.

\subsection{The $[\mathrm{OIII}]$ and $\mathrm{H} \beta$ line emission}

The medium ionization line of $[\mathrm{O}$ III $] 5007 \AA$ (Fig. 2) displays a different morphology than the lower ionization lines of e.g. $\mathrm{H} \alpha+[\mathrm{N} \mathrm{II}]$ or $[\mathrm{S} \mathrm{II}]$. The two arcs (area I) are present but their emission is very weak. Another filamentary structure is detected in the north, at $\alpha=19^{\mathrm{h}} 59^{\mathrm{m}} 13^{\mathrm{s}}$ and $\delta=34^{\circ} 12^{\prime} 30^{\prime \prime}$ (area Ia), where the arc of area I merges with the background. Emission from area II is much better defined than in its $\mathrm{H} \alpha+[\mathrm{N}$ II] or [S II] counterparts. We now observe a $\sim 23^{\prime}$ long filamentary structure characterized by a projected thickness of $\sim 40^{\prime \prime}$.

Interestingly, the composite structure in area III looks quite different in this emission line. We observe two thin filaments at the north and south boundaries of this area separated by diffuse emission, while contrary to the low ionization images a $\sim 11^{\prime}$ long tail of diffuse emission is detected to the east. A new structure is also detected in [O III $]$ to the south-east of this tail at $\alpha \simeq 20^{\mathrm{h}} 02^{\mathrm{m}} 08^{\mathrm{s}}$ and $\delta \simeq 33^{\circ} 47^{\prime} 47^{\prime \prime}$ without any obvious counterpart in $\mathrm{H} \alpha+[\mathrm{N} I \mathrm{II}]$ or $[\mathrm{S} \mathrm{II}]$. Diffuse emission is only present in the east half of area IV which would be very difficult to identify if it were not for the $\mathrm{H} \alpha+[\mathrm{N} \mathrm{II}]$ image.

The $\mathrm{H} \beta$ line emission image resembles the morphology of the $\mathrm{H} \alpha+[\mathrm{N}$ II $]$ image but at a much lower intensity level and is not shown here. The two arcs in area I are barely seen, while the long filament in area II is less well defined. Emission from areas III and IV is traced further to the north where the emission from the latter area is very weak.

\subsection{The $[\mathrm{OII}]$ line emission}

The $[\mathrm{O}$ II $] 3727 \AA$ low ionization line image best describes the newly detected structures (Fig. 3). Emission from the two arcs in area $\mathrm{I}$ is detected as well as emission from the filament in area Ia. The long [O III] filament in the south (area II) is also present in [O II] but its extent is shorter $\sim 16^{\prime}$, while its projected width is $\sim 34^{\prime \prime}$. The south and north boundaries of the structure in area III are very bright with diffuse emission between them. Especially, the 


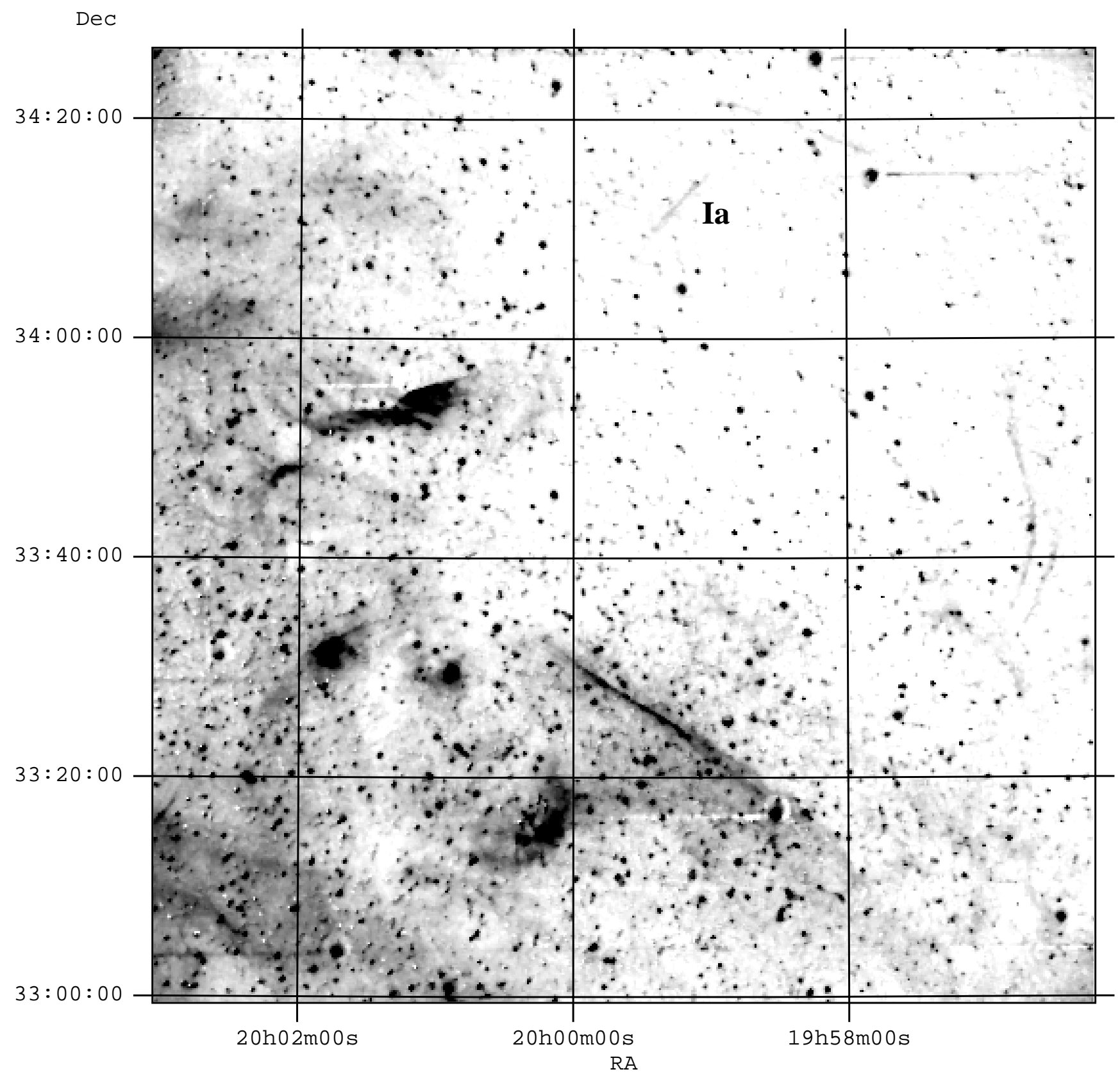

Fig. 2. The neighbourhood around the candidate remnant in the [O III] filter. The image has been smoothed to suppress the residuals from the imperfect continuum subtraction, while the shadings run linearly from 0.0 to $20 \times 10^{-17} \mathrm{erg} \mathrm{s}^{-1} \mathrm{~cm}^{-2}$ arcsec ${ }^{-2}$. Emission from areas I and Ia is barely seen in the west and in the north, respectively, but is definitely detected.

north boundary is very sharp. We measure a decrease in flux by a factor of $\sim 3.5$ in only 3 pixels, i.e. $\sim 15^{\prime \prime}$.

\section{The spectral observations}

Long-slit spectra were obtained from the peculiar structures discovered in the north-east neighbourhood of CTB 80. The approximate slit locations are shown in Fig. 3 as long rectangles in the north-south direction, while the equatorial coordinates of the slit centers are given in Table 3 . The red lines of $\mathrm{H} \alpha,[\mathrm{N}$ II] and [S II] are accurately established, while the bluer lines of $\mathrm{H} \beta$, and [O III] are almost always present with a range of significance. The fluxes of the individual lines are given in Table 4 along with their signal to noise ratio as calculated by the relevant IRAF routines. Consequently, these signal to noise values do not include calibration errors which for the specific observations are, on the average, $\sim 6 \%$. Aperture spectra extracted from the north of the corresponding slit center are designated as IIIn or IVn, while those extracted from the south are designated as IIIs or IVs (see also the caption of Table 4). Common to all spectra is the strong sulfur emission relative to $\mathrm{H} \alpha$, indicative of shock heated gas. In addition, the ratio of the sulfur lines is close to the low density limit of $\sim 1.4-1.5$ (e.g. Osterbrock 1989), characteristic of spectra from evolved supernova remnants. 
Table 4. Relative line fluxes from long-slit spectra.

\begin{tabular}{|c|c|c|c|c|c|c|}
\hline & area I & area II & 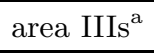 & area $\operatorname{IIIn}^{\mathrm{a}}$ & area $I V n^{b}$ & area $\mathrm{IVs}^{\mathrm{b}}$ \\
\hline \multicolumn{7}{|l|}{ Line $(\AA)$} \\
\hline $4861 \mathrm{H} \beta$ & $10^{\mathrm{c}}(5)^{\mathrm{d}}$ & $21(11)$ & $23(17)$ & $17(4)$ & $16(3)$ & $17(5)$ \\
\hline 4959 [O III] & - & $23(22)$ & $8(11)$ & $33(8)$ & - & - \\
\hline 5007 [O III] & $17(7)$ & $68(45)$ & $24(22)$ & $120(30)$ & $17(3)$ & $38(2)$ \\
\hline $6548[\mathrm{~N} \mathrm{II}]$ & $32(14)$ & $20(22)$ & $23(41)$ & $18(13)$ & $27(5)$ & $22(12)$ \\
\hline $6563 \mathrm{H} \alpha$ & $100(29)$ & $100(109)$ & $100(120)$ & $100(50)$ & $100(19)$ & $100(41)$ \\
\hline $6584[\mathrm{~N}$ II] & $102(31)$ & $66(73)$ & $73(110)$ & $67(38)$ & $87(17)$ & $71(33)$ \\
\hline 6716 [S II] & $55(22)$ & $35(45)$ & $66(105)$ & $48(26)$ & $74(18)$ & $61(30)$ \\
\hline $6731[\mathrm{~S} \mathrm{II}]$ & $39(18)$ & $25(32)$ & $46(97)$ & $32(16)$ & $51(13)$ & $44(22)$ \\
\hline Absolute $\mathrm{H} \alpha^{\mathrm{e}}$ & 6.7 & 22.0 & 63.0 & 14.7 & 12.8 & 15.6 \\
\hline $\mathrm{H} \alpha / \mathrm{H} \beta$ & $10(5)$ & $4.8(11)$ & $4.4(16)$ & $5.8(4)$ & $6.3(2.5)$ & $6.1(5)$ \\
\hline$[\mathrm{O} \mathrm{III}] / \mathrm{H} \beta$ & $1.7(5)$ & $3.2(11)$ & $1.0(22)$ & $7.1(4)$ & $1.1(3)$ & $2.2(2)$ \\
\hline$[\mathrm{S} \mathrm{II}] / \mathrm{H} \alpha$ & $0.9(20)$ & $0.6(48)$ & $1.1(92)$ & $0.8(25)$ & $1.2(16)$ & $1.0(20)$ \\
\hline$[\mathrm{S}$ II $] /[\mathrm{S} \mathrm{II}]$ & $1.4(14)$ & $1.4(26)$ & $1.4(71)$ & $1.5(14)$ & $1.5(10)$ & $1.4(18)$ \\
\hline
\end{tabular}

a Center of aperture IIIs is $55^{\prime \prime}$ south of slit center, while IIIn is $22^{\prime \prime}$ north.

b Center of aperture IVn is $22^{\prime \prime}$ north of slit center, while IVs is $45^{\prime \prime}$ south.

${ }^{\mathrm{c}}$ Uncorrected for interstellar extinction.

$\mathrm{d}$ The signal to noise of the quoted numbers is given in the parentheses.

e The absolute $\mathrm{H} \alpha$ flux is given in units of $10^{-17} \mathrm{erg} \mathrm{s}^{-1} \mathrm{~cm}^{-2} \operatorname{arcsec}^{-2}$.

All fluxes normalized to $F(\mathrm{H} \alpha)=100$.

The medium ionization line of $[\mathrm{O} \mathrm{III}]$ is present in all spectra. However, its strength relative to $\mathrm{H} \alpha$ varies significantly. We measure a weak [O III] emission in area IVn amounting to $17 \%$ of the $\mathrm{H} \alpha$ emission, while the maximum [O III] emission is observed in area II where it amounts to $68 \%$ of the $\mathrm{H} \alpha$ emission (Table 4).

\section{Observations at other wavelengths}

SNRs often produce emission in other bands of the electromagnetic spectrum, the characteristics of which may provide clues to the nature of the object. In particular, nonthermal radio emission is an almost certain indicator of shock acceleration in the blast wave of a SNR.

\subsection{Radio}

We have looked for radio observations of suitable resolution and sensitivity which might have detected emission from the new candidate remnant, and find: a $6 \mathrm{~cm}$ survey made with the Greenbank $91 \mathrm{~m}$ telescope (Condon et al. 1994), the $11 \mathrm{~cm}$ Galactic plane survey made by Reich et al. (1990), and the $92 \mathrm{~cm}$ WENSS survey (Rengelink et al. 1997). All three data sets are publicly available from various servers on the internet. In addition, we have reanalyzed a Westerbork $92 \mathrm{~cm}$ synthesis map of the adjacent object CTB 80 , since its field of view does include the field observed in the optical, albeit with somewhat attenuated intensities. We will consider these data sets in order of increasing wavelength.

The $6 \mathrm{~cm}(4.85 \mathrm{GHz})$ northern sky survey was made with the $91 \mathrm{~m}$ Greenbank meridian transit telescope (Condon et al. 1994) at an angular resolution near 3!5. The total estimated noise (thermal plus confusion) for the galactic longitude of $l=70^{\circ} .1$ is under $4 \mathrm{mJy} \mathrm{beam}^{-1}$, although examination of this low latitude field itself suggests that there may be weak, diffuse emission from the Galaxy, increasing the confusion level. Condon et al. (1994) note that the reduction technique used will tend to suppress emission extended by more than $20^{\prime}$ in declination, and this could affect the proposed candidate remnant with its overall size of about $1^{\circ}$.

The radio emission is dominated by the extended sources associated with the bright HII regions Sh 2-99/100 (Sharpless 1959) to the southeast, and the SNR CTB 80, to the southwest. Nonetheless, radio contours overlaid on the optical nebulosity (Fig. 4) do show faint emission with fairly good morphological correspondence to filaments I and IV. There may also be faint emission from II, but its proximity to the strong emission from Sh 2-99/100 means that its possible detection should be treated with caution. The faint feature near Ia bears little resemblence to the narrow optical filament. No emission is detected from the position of filament III. The rather strong emission from the filaments of LBN 156 comes from an extension of the northern arc of CTB 80, and may not be related to this candidate remnant. All of the $6 \mathrm{~cm}$ radio emission which 


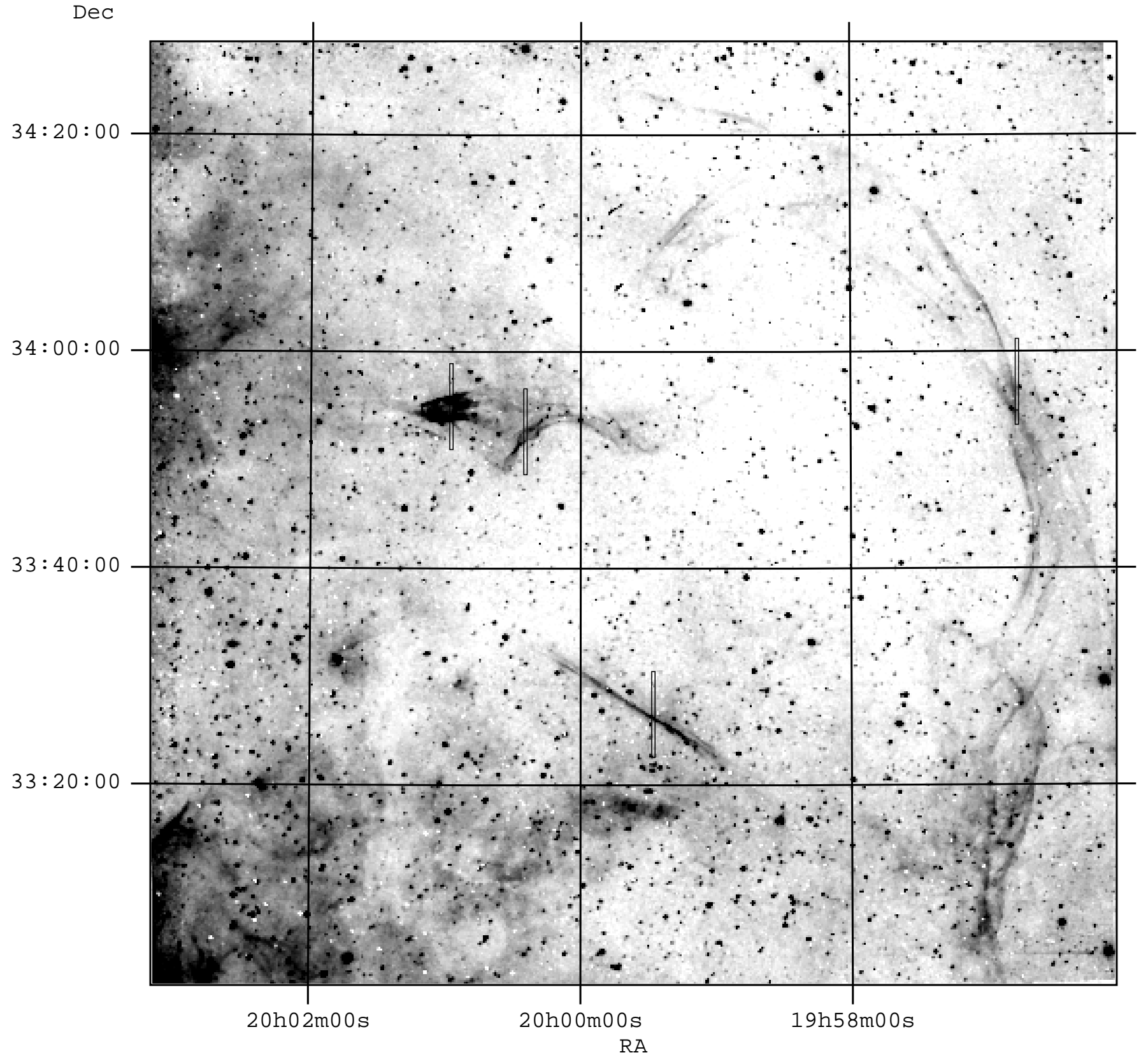

Fig. 3. The $[\mathrm{OII}]$ image clearly delineates the new structures detected in this field. The shadings run linearly from 0.0 to $50 \times 10^{-17} \mathrm{erg} \mathrm{s}^{-1} \mathrm{~cm}^{-2} \operatorname{arcsec}^{-2}$ and the image has been smoothed to suppress the residuals from the imperfect continuum subtraction. The slit locations are shown as long rectangles.

might be associated with the SNR is very weak, with typical surface brightnesses of $5-10 \mathrm{mJy}_{\text {beam }}{ }^{-1}$. These can be considered marginal detections at best; only the morphological similarity to filaments I, IV, and perhaps II, indicates that the emission is likely to be genuine.

We next considered the $11 \mathrm{~cm}(2.695 \mathrm{GHz})$ galactic plane survey made with the Effelsberg $100 \mathrm{~m}$ telescope (Reich et al. 1990). The angular resolution was 4.3 , with an expected noise level near $5 \mathrm{mJy}_{\text {beam }}{ }^{-1}$. In our analysis of a map obtained from the MPI website we were able to locate emission similar to that at $6 \mathrm{~cm}$ near filaments I, Ia and IV. The strength is typically 10-15 mJy beam ${ }^{-1}$, although the emission from IV (which falls on a gradient increasing to the east) may be stronger than this. The emission associated with filament I (Fig. 5) is the most isolated, with a typical brightness of between 9 and 16 mJy beam ${ }^{-1}$ (to be compared with 3 $14 \mathrm{mJy}$ beam $^{-1}$ at $6 \mathrm{~cm}$ ). We conclude that the radio emission is probably nonthermal, supporting our interpretation that the detected filaments are part of a SNR.

The WENSS maps (Rengelink et al. 1997) for this region could not be used to image extended emission because of the relative proximity of the strong source Cyg A, and have not been considered further. However, we did analyze a $92 \mathrm{~cm}$ map centered on the well-known SNR CTB 80, with a resolution of about $0.9^{\prime} \times 1.6^{\prime}$, and a sensitivity 


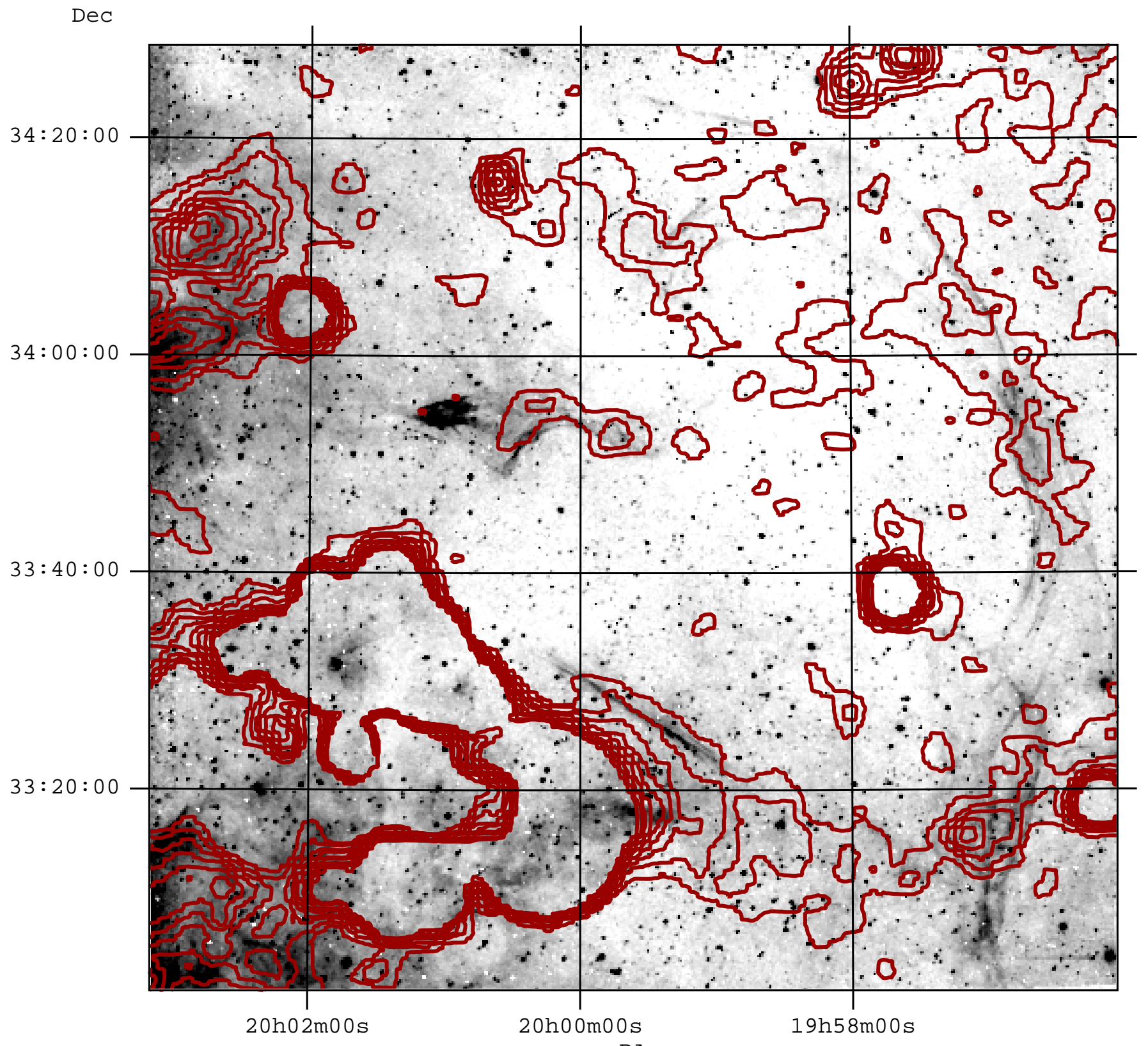

RA

Fig. 4. The radio $4850 \mathrm{MHz}$ contours (Condon et al. 1994) overlaid on the [O II] image shown in Fig. 3. The radio contours scale linearly from 0.002 to $0.06 \mathrm{Jy} /$ beam by $0.00725 \mathrm{Jy} /$ beam. Strong, extended radio emission is seen in the south-west, while faint radio emission seems correlated with the newly detected structures.

near $5 \mathrm{mJy}^{\mathrm{beam}}{ }^{-1}$. Although of good quality, there are faint remnants of grating lobes from many extended galactic sources, and the distant source Cyg A. The extended radio emission near filament Ia is clearly present, and there is a hint of the emission arc from I, although it is crossed by a distant grating lobe from Cyg A. There are also large-scale gradients crossing the map, which make the determination of the intensity of faint features uncertain. A brightness estimate of some of the extended emission near Ia, when compared with the $6 \mathrm{~cm}$ emission, gives a spectral index of, $\alpha \simeq-1$, once again consistent with nonthermal emission.

\subsection{Other wavebands}

Emission might also be expected in the infrared and X-ray bands. We have inspected maps from the IRAS survey in the four bands $(12,25,60$ and $100 \mu \mathrm{m})$, but there are no obvious features which might suggest an interaction of the candidate supernova remnant shell with the source of IR emission like e.g. in CTB 80 (Fesen et al. 1988). There is a depression seen at all four bands which closely matches the western edge of the shell, extending from the southern end of filament I. Whether it is physically associated, or just a chance coincidence, is difficult to say; it is the region of lowest IR emission in the region. 
A perusal of X-ray data available reveals no obvious associated emission features. The $3 \sigma$ upper limit, calculated from photons collected in $\sim 430 \mathrm{~s}$ from the ROSAT All-sky survey, close to this area, is $\sim 6 \times 10^{-4}$ cts s$^{-1}$ arcmin $^{-2}$ in the $0.1-2.0 \mathrm{keV}$ band.

\subsection{A hypothesis}

The optical observations revealed the presence of filamentary structures which display the characteristic signature of emission from shock heated gas. In addition, faint radio emission seems spatially correlated with almost all of these new structures. We propose that the emission from areas I, II, and IV belongs to a single expanding supernova remnant shell. The observed variations in the absolute line fluxes could be due to density variations of the interstellar "clouds" leading to shock velocity variations, or due to intrinsic absorption or due to possible abundance variations. The relation of the network of filaments seen in area III to the candidate remnant is not clear but cannot be ruled out at the moment. The estimated center of this candidate remnant is located at a galactic longitude of $70^{\circ} 1$ and a galactic latitude of 2.3 . The angular radius is $\sim 42^{\prime}$ corresponding to $\sim 24 \mathrm{pc}$ at a distance of $2 \mathrm{kpc}$ (see Sect. 6.1). Further radio spectral observations would be required to establish the non-thermal nature of the emission from these structures and determine the extent to which they are related.

\section{Discussion}

Several new emission line structures, as described above, have been discovered to the north-east of the supernova remnant CTB 80. Even though their average absolute fluxes span a wide range of values, their sulfur line emission is strong relative to $\mathrm{H} \alpha$, as would be expected for emission from a supernova remnant.

\subsection{Area I}

The two filamentary arcs to the north of LBN 156 are very faint but clearly visible, especially in the $[\mathrm{O} \mathrm{II}] \mathrm{im}$ age (Fig. 3). The angular extent of these filaments covers roughly more than a quarter of a circle's circumference. In addition, the faint filamentary emission detected at position Ia (Fig. 4) seems to be related to the long arcs and is probably projected at the specific location. The [O III] image also shows weak emission in areas I and Ia, while the absolute amount of flux in this line is comparable to the $\mathrm{H} \beta$ flux suggesting the presence of complete recombination zones behind the shock front (Raymond et al. 1988). The strong $[\mathrm{O} \mathrm{II}]$ emission relative to the weaker [O III] emission indicates a shock velocity in the range of 90$120 \mathrm{~km} \mathrm{~s}^{-1}$ and/or a partially neutral preshock medium (Cox \& Rayond 1985).

Deep long-slit spectra taken at area I provide us with more information about the physical properties at the specified location. The total sulfur line flux amounts to
$90 \%$ of the $\mathrm{H} \alpha$ flux identifying it as emission from shock heated gas. The $[\mathrm{O} I I I]$ flux is only $\sim 2$ times higher than the $\mathrm{H} \beta$ flux, while the latter is a factor of $\sim 10$ weaker than the $\mathrm{H} \alpha$ flux. This implies a significant attenuation of the optical emission due to interstellar absorption. Adopting the interstellar reddening curve of Whitford (1958) as presented by Kaler (1976), we find a logarithmic interstellar extinction, $c=1.6 \pm 0.3$ towards area I. The computerized model of Hakkila et al. (1997) on the visual interstellar extinction allows us to obtain a very rough estimate on the distance to area I. Use of this code shows that distances greater than $2 \mathrm{kpc}$ are compatible with the measured extinction of 1.6 and the following results will be scaled to this distance.

The observed angular radius of $42^{\prime}$ is equivalent to $24.2 D_{2 \mathrm{kpc}} \mathrm{pc}$, while a typical projected FWHM of the filaments in the north-west is $0.15 D_{2 \mathrm{kpc}}$ pc. (Where $D_{2 \mathrm{kpc}}$ is the distance to the filaments in units of $2 \mathrm{kpc}$.) The observed sulfur line ratio approaches the low density limit but given the statistical uncertainties we estimate that the actual electron density is less than $\sim 160 \mathrm{~cm}^{-3}$ at a $2 \sigma$ confidence.

An estimate of the preshock cloud density can be made through the use of the equation given by Fesen \& Kirshner (1980) which relates the electron density, the preshock cloud density and the shock velocity. Given the above upper limit on the electron density and the range of shock velocities, we expect preshock cloud densities less than $\sim 4 \mathrm{~cm}^{-3}$. For this density of $4 \mathrm{~cm}^{-3}$, we find that the energy of the explosion $E$ should lie in the range of $0.8^{-}$ $1.6 \times 10^{51} D_{2 \mathrm{kpc}}^{3}$ erg (Hailey \& Craig 1994). Since the derived preshock cloud density is only an upper limit, the energy $E$ should be less than $1.6 \times 10^{51} D_{2 \mathrm{kpc}}^{3}$ erg.

The radio emission found, though weak and certainly in need of confirmation, appears to support our suggestion that observed optical emission originates from a SNR. There is little evidence for fine-scale structure. Indeed, the $92 \mathrm{~cm}$ maps show no evidence for emission features as narrow as the optical filaments, as is seen in some SNRs.

The nondetection of X-ray emission is not too surprising, as soft X-rays can be readily absorbed by the ISM, and the emission might be intrinsically weak. As for the "hole" in the infrared background, while it might be fortuitous, similar features have been noted in association with other SNRs. For example, Braun \& Strom (1986) find a cavity associated with the Cygnus Loop.

\subsection{Area II}

In the southern areas of our field we have discovered a long, relatively thin structure which is characterized by very strong oxygen emission both in the low and medium ionization images (Figs. 2 and 3, Table 4). The sulfur to $\mathrm{H} \alpha$ line ratio is 0.6 , a value which is not far away from the limiting value of $0.4-0.5$ used for distinguishing between H II regions and shock heated gas (Fesen et al. 1985). In favor of the latter case is the fact that the spectra taken 
to the south and north of the [O III] filament, i.e. into the diffuse emission, are characterized by a $[\mathrm{S} \mathrm{II}] / \mathrm{H} \alpha$ ratio of $\sim 0.4$.

In the case where the observed emission originates indeed from shock heated gas, we estimate a shock velocity around $100 \mathrm{~km} \mathrm{~s}^{-1}$ and an electron density close to the low density limit. Note that a spectrum from a planar shock propagating at $\sim 110 \mathrm{~km} \mathrm{~s}^{-1}$ with equilibrium ionization matches, acceptably well, the observations (Hartigan et al. 1987). The authors have also constructed shock models with complete preionization. The model that most closely approximates the observations corresponds to a shock velocity of $\sim 80 \mathrm{~km} \mathrm{~s}^{-1}$, and probably less, even though the calculated sulfur line flux is less than that observed. The presence of radio emission along the [O III] filament may suggest their physical association but it is only the determination of its non-thermal nature that would firmly establish this proposition.

\subsection{Area III}

The new peculiar source of emission exhibits the highest surface brightness among the rest of the new structures in the field, with very strong sulfur line emission (Table 4). Spectra extracted from different apertures along the slit indicate a $[\mathrm{S} \mathrm{II}] / \mathrm{H} \alpha$ ratio of $0.8-1.1$, characteristic of emission from shock heated gas. The difference seen in the low and medium ionization images is also evident in the results from the long-slit spectra reported in Table 4, where substantial variations of the [O III] flux are observed. In addition, the calibrated images suggest that the [O II] flux is much stronger than the [O III] flux in area IIIs than in area IIIn. These observational constraints cannot be met by the equilibrium ionization models of Hartigan et al. (1987). Better agreement is achieved by the complete preionization models.

The $60 \mathrm{~km} \mathrm{~s}^{-1}$ model predicts an [O III] flux comparable to the $\mathrm{H} \beta$ flux, while the [O II] flux is several times stronger than the [O III] flux. This is similar to what we observe in area IIIs. However, the spectrum from area IIIn exhibits an [O III] flux which is $\sim 6$ times stronger than the $\mathrm{H} \beta$ flux, while at the same location the [O II] flux is only 2 times stronger than the [O III] flux. The $80 \mathrm{~km} \mathrm{~s}^{-1}$ complete preionization model of Hartigan et al. (1987) shows similar characteristics. We note here that the model calculations were performed for a preshock density of $100 \mathrm{~cm}^{-3}$, while our long-slit spectra and the estimates of the shock velocity suggest that the preshock cloud density should not exceed a few nuclei per $\mathrm{cm}^{-3}$. Calculations focusing on the specific problem under study would probably be more favorable since we are in the range of velocities that dramatically affect the [O III] flux.

The strong oxygen emission from feature III reminds one of the oxygen-rich filaments which have been observed in a small number of other remnants. Although usually associated with young objects (Van den Bergh 1988), one of the protrusions seen in the $\sim 10000$ yr old Vela supernova

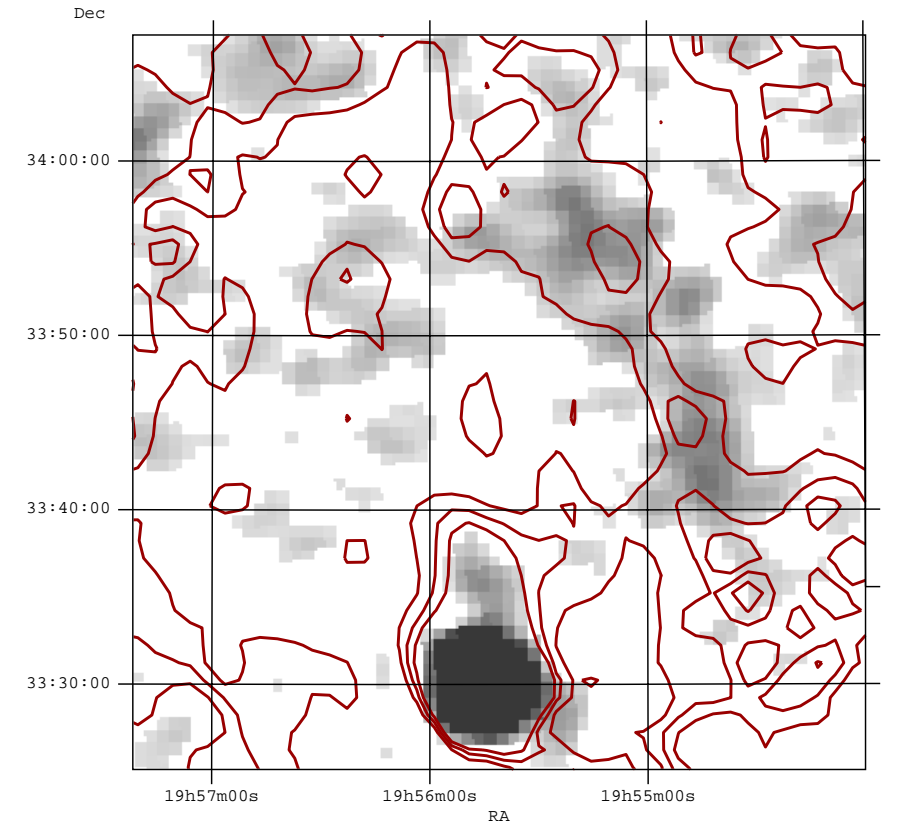

Fig. 5. The radio emission from Area I at $4850 \mathrm{MHz}$ is shown in grey scale. The contours represent the $11 \mathrm{~cm}$ emission detected in the Effelsberg survey (Reich et al. 1990) and the contour levels are set at 8,16 and $32 \mathrm{mJy}$ beam $^{-1}$.

remnant (Aschenbach et al. 1995; referred to as D) is also oxygen rich. Indeed the wedge shape of III (Fig. 3), and its apparent location near the rim of the candidate remnant, are also features which it shares with D.

\subsection{Area IV}

The fourth new structure detected in this part of the sky has a semi-circular shape and is a weak source of forbidden line radiation. The morphology of this source is mainly diffuse and patchy but yet, the flux emitted in the sulfur lines is stronger than that emitted in the $\mathrm{H} \alpha$ line. Spectra extracted from different apertures along the slit provide evidence for emission from shock heated gas. The interstellar extinction is not accurately determined due to the low confidence of the $\mathrm{H} \beta$ flux. In favor of a supernova remnant association of the optical emission from this area is the radio emission seen in Fig. 4, where its morphology seems to match that of the optical. However, it is clear that the non-thermal nature of the radio emission must be established before one can accept such an association.

\section{Conclusions}

Deep imaging and spectral observations have revealed unknown filamentary and diffuse structures to the north-east of the well known supernova remnant CTB 80. The long, faint filaments in area I are compatible with emission from the cooling zone behind a $90-120 \mathrm{~km} \mathrm{~s}^{-1}$ shock propagating into a medium characterized by preshock densities of a few nuclei per $\mathrm{cm}^{-3}$. 
We propose that the optical emission from areas I, Ia, II and IV belongs to a single expanding shell of an unknown remnant. Although emission from shock heated gas is also observed from region III, its relation to the new candidate remnant is less certain. Radio spectral observations are needed to test our suggestions.

Acknowledgements. We would like to thank J. Papamastorakis, J. Ventura and E. V. Palaiologou for their useful comments. We would also like to thank the referee B. Aschenbach whose remarks helped clarify the scope of this paper. Skinakas Observatory is a collaborative project of the University of Crete, the Foundation for Research and Technology-Hellas and the Max-Planck-Institut für Extraterrestrische Physik. This work has been supported by a P.EN.E.D. program of the General Secretariat of Research and Technology of Greece. The Westerbork Synthesis Radio Telescope is operated by ASTRON with financial support from the Netherlands Organization for Scientific Research (NWO). This research has made use of data obtained through the High Energy Astrophysics Science Archive Research Center Online Service, provided by the NASA/Goddard Space Flight Center.

\section{References}

Aschenbach, B., Egger, R., \& Trümper, J. 1995, Nature, 373, 587

Braun, R., \& Strom, R. G. 1986, A\&A, 164, 208
Condon, J. J., Broderick, J. J., Seielstad, G. A., Douglas, K., \& Gregory, P. C. 1994, AJ, 107, 1829

Cox, D. P., \& Raymond, J. C. 1985, ApJ, 298, 651

Fesen, R. A., \& Kirshner, R. P. 1980, ApJ, 242, 1023

Fesen, R. A., Blair, W. P., \& Kirshner, R. P. 1985, ApJ, 292, 29

Fesen, R. A., Shull, J. M., \& Saken, J. M. 1988, Nature, 334, 229

Hartigan, P., Raymond, J., \& Hartmann, L. 1987, ApJ, 316, 323

Hakkila, J., Myers, J. M., Stidham, B. J., \& Hartmann, D. H. 1997, AJ, 114, 2043

Hailey, C. J., \& Craig, W. W. 1994, ApJ, 434, 635

Kaler, J. B. 1976, ApJS, 31, 517

Lynds, B. T. 1965, ApJS, 12, 163

Mavromatakis, F., Ventura, J., Paleologou, E. V., \& Papamastorakis, J. 2001, A\&A, 371, 300

Osterbrock, D. E. 1989, Astrophysics of gaseous nebulae (W. H. Freeman \& Company)

Parker, R. A. R., Gull, T. R., \& Kirshner, R. P. 1979, An emission-line survey of the Milky Way, NASA SP-434

Raymond, J. C., Hester, J. J., Cox, D., et al. 1988, ApJ, 324, 869

Reich, W., Fürst, E., Reich, P., \& Reif, K. 1990, A\&A, 85, 633

Rengelink, R. B., Tang, Y., de Bruyn, A. G., et al. 1997, A\&AS, 124,259

Sharpless, S. 1959, ApJS, 4, 257

Van den Bergh, S. 1988, ApJ, 327, 156

Wheelock, S. L., Gautier, T. N., Chillemi, J., et al. 1994, IRAS sky survey atlas, Explanatory supplement

Whitford, A. 1958, AJ, 63, 201 